\title{
Experimental demonstration of a wavelength demultiplexer based on negative-refractive photonic-crystal components
}

\author{
Takashi Matsumoto, Tomohiko Asatsuma, and Toshihiko Baba ${ }^{a}$ \\ Department of Electrical and Computer Engineering, Yokohama National University, 79-5 Tokiwadai, \\ Hodogayaku, Yokohama 240-8501, Japan
}

(Received 3 July 2007; accepted 14 August 2007; published online 30 August 2007)

\begin{abstract}
Negative refraction of light was observed at near-infrared wavelengths in a silicon-on-insulator photonic-crystal-slab superprism having low-loss interface structures. It was used for wavelength demultiplexing as a diffraction grating, in combination with a photonic-crystal superlens used for focusing light beams. Coarse wavelength demultiplexing action with a channel spacing of 7-27 nm was demonstrated in a device whose size was only $80 \times 100 \mu \mathrm{m}^{2}$ (excluding input and output waveguides). These results agree well with those obtained by a finite-difference time-domain simulation. () 2007 American Institute of Physics. [DOI: 10.1063/1.2779927]
\end{abstract}

Photonic crystals (PCs) can be used to produce negative refraction of light. This phenomenon is actually a form of diffraction that arises from the anomalous dispersion characteristics of PCs having multidimensional periodic structures. The superprism effect is one of the unique phenomena associated with negative refraction. ${ }^{1-9}$ A superprism has an angular dispersion that far exceeds those of conventional prisms. Therefore, superprisms were anticipated to improve the wavelength resolution and/or reduce the device size of wavelength demultiplexers (DEMUXs). However, we demonstrated theoretically that such a DEMUX cannot be simply realized since the deflected light beam diverges in superprisms, particularly in the strongly dispersive condition for high-order photonic bands. ${ }^{10}$ To remove this constraint, we proposed integrating a PC superlens with a superprism. ${ }^{11} \mathrm{~A}$ PC superlens collects and focuses a diverging light beam by negative refraction. ${ }^{12-24}$ We clearly observed focusing of near-infrared light in a PC with a flat surface. ${ }^{24} \mathrm{~A}$ superlens forms a symmetric image of an object with respect to the surface of the PC since the focusing mechanism is completely different from that of conventional lenses. This means that multiple light beams are focused in parallel so that their optical paths do not intersect each other. This property can be used to effectively compensate the degradation in the wavelength resolution caused by the divergence of demultiplexed light beams. As alternative solution to this problem, Momeni et al. recently proposed a DEMUX, which utilizes the band edge of the lowest-order photonic band simultaneously exhibiting a similar angular dispersion to our proposed solution and convergence of light beams. ${ }^{25}$ However, these two combined properties restrict the design flexibility of the PC. A long preconditioning region must be inserted between the input waveguide and the PC so that the beam separation length and the focal length are equal. In contrast to this, in our device, these two lengths can be optimized independently of each other without inserting a preconditioning region, allowing the total device size to be about ten times smaller. In a previous study, we theoretically simulated the operation of our device using finite-difference time-domain (FDTD) method. ${ }^{11}$ In this present study, we experimentally demonstrated its operation. First, we investigated negative

\footnotetext{
${ }^{a)}$ Electronic mail: baba@ynu.ac.jp
}

refraction in a PC superprism whose surface structure had been optimized to minimize unwanted reflection and scattering losses. Then, we fabricated a DEMUX device designed to have a wide channel spacing of approximately $10 \mathrm{~nm}$, and observed its operation at near-infrared wavelengths.

In the fabrication, we prepared a silicon-on-insulator (SOI) substrate that had a $0.26-\mu$ m-thick Si layer and a 1- $\mu$ m-thick $\mathrm{SiO}_{2}$ layer. We formed the PC pattern using e-beam lithography and $\mathrm{SF}_{6}$ inductively coupled plasma etching, and the air-bridge structure using HF wet etching. Figure 1(a) shows the fabricated PC; it consists of airholes in a square lattice rotated by $45^{\circ}$. The lattice constant $a$ was $0.47 \mu \mathrm{m}$ and the airhole diameter $2 r$ was $0.29 \mu \mathrm{m}$ (i.e., $2 r / a=0.62)$. Figure $1(\mathrm{~b})$ shows the calculated dispersion surface of the second-order photonic band that was used for both the superprism and the superlens. In this calculation, we assumed in-plane polarization (i.e., TE polarization), $2 r / a$ $=0.624$, and that the equivalent modal index of the slab was 2.963. For the superprism, the angle of incidence was fixed at $10^{\circ}$ to the normal of the PC surface. In general, the Poynting vector $S$ in the PC is determined by the gradient of the equifrequency contours. The direction of the Poynting vector $S_{p}$ of the superprism changes with frequency, as indicated by

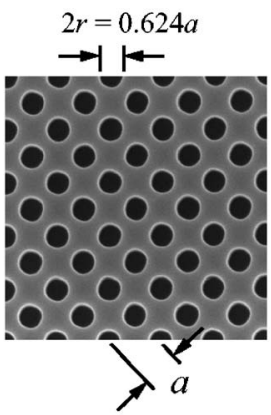

(a)

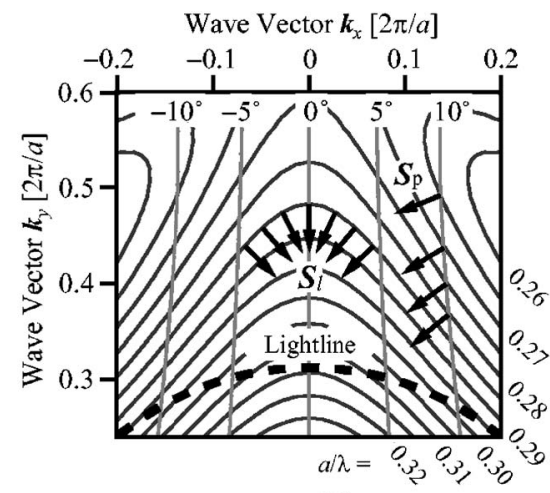

(b)
FIG. 1. Fundamental structure of PC and dispersion surface. (a) Scanning electron microscope (SEM) image of fabricated PC. (b) Part of the dispersion surface calculated using two-dimensional plane-wave expansion method. Dark gray curve and light gray curve denote equifrequency and equi-incident angles, respectively. $\boldsymbol{S}_{p}$ and $\mathbf{S}_{l}$ are Poynting vectors used for superprism and flat lens, respectively. 


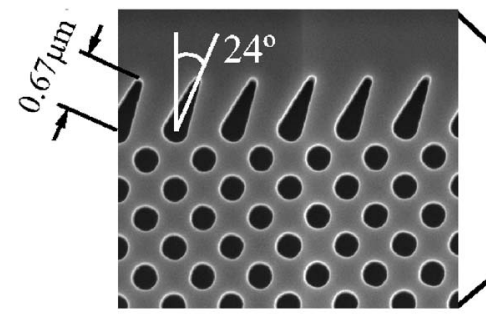

(a)

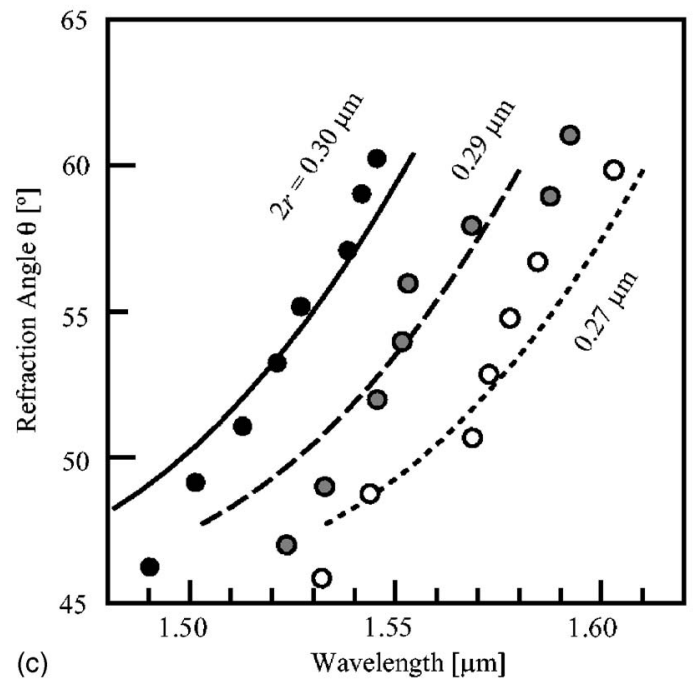

FIG. 2. (Color online) Superprism effect. (a) SEM image of fabricated PC superprism with tapered airhole interface. (b) NFP of refracted light in the superprism. (c) Dispersion of refraction angle observed for three samples with different $2 r$. Circles denote experimental results and curves theoretical ones calculated in a FDTD simulation.

the black arrows on the equi-incident angle curve of $10^{\circ}$ in Fig. 1(b).

Tapered airholes angled at $24^{\circ}$ in the opposite direction to the incident angle were fabricated at the input surface of the PC superprism, as shown in Fig. 2(a). This interface structure functions as a phase converter for the incident light so that it becomes a Bloch wave in the PC and effectively reduces the reflection and scattering losses. ${ }^{26}$ Indeed, this interface structure is very important for clearly observing negative reflection since incident light is almost completely reflected when the PC surface is terminated by normal circular airholes. In the measurement, TE-polarized light from a tunable laser was coupled to a 0.5 - $\mu \mathrm{m}$-wide single-mode $\mathrm{Si}$ wire waveguide, which was integrated on the same substrate as the PC. Light guided through this waveguide was then expanded by a $2.3-\mu \mathrm{m}$-wide parabolic taper that was used to control the beam divergence and it was then incident on the PC. The wavelength $\lambda$ of the light was first tuned to $1.37 \mu \mathrm{m}$, which satisfies the condition for leakage for the guided light (i.e., the light cone condition). The near-field pattern (NFP) of light propagation in the PC was observed from above the PC using InGaAs camera. Figure 2(b) shows an image obtained using this setup; it clearly shows negative refraction. On the other hand, similar images could not be observed for $\lambda>1.39 \mu \mathrm{m}$, outside of the light cone condition. Even in this case, negative refraction was confirmed from light scattering at the end of the PC. Since the scattered light extended over a width of $10 \mu \mathrm{m}$, the refraction angle was estimated after the light had been focused by the PC superlens, in a manner similar to the case for the DEMUX device, as described below. Figure 2(c) shows the dispersion of the refraction angle $\theta$ for three PCs with different $2 r$. The refraction angle varied in the range of $46^{\circ}-61^{\circ}$ for a wavelength variation of $60 \mathrm{~nm}$. The wavelength range shifts to longer wavelengths as the airholes become smaller. This is a reasonable result since the equifrequency contours shift to lower frequencies when the lattice constant is kept fixed and the airholes are made smaller. The experimental plots agree well with theoretical curves obtained using the FDTD method.

Figure 3(a) shows the fabricated DEMUX device. Here, a Si-wire waveguide with a parabolic taper was again used as the input waveguide. Light was tilted at $10^{\circ}$ relative to the normal and incident on the superprism that had parallel surfaces. The PC had the same design as that described above. The same tapered airholes were used at the input and output surfaces. Output light from the superprism has the same angle as the incident angle, since the tangential component of the incident wave vector is conserved. Light beams having different wavelengths are spatially separated in the superprism and are incident at different positions on the superlens. The superlens was tilted at an angle of $10^{\circ}$ relative to the superprism so that the output light from the superprism is oriented normal to the flat surface of the superlens. The PC for the superlens was slightly modified from that described above such that $a=0.51 \mu \mathrm{m}$ and $2 r=0.31 \mu \mathrm{m}$ to adjust the operating wavelength to $\lambda \sim 1.55 \mu \mathrm{m}$, while maintaining a dispersion surface similar to that shown in Fig. 1(b). As indicated by the black arrows in Fig. 1(b), the Poynting vectors $S_{l}$ of the superlens are oriented toward focusing directions. Similarly to the case of the superprism, tapered airholes were used at the input surface to minimize reflection and scattering losses. ${ }^{24}$ The output surface of the superlens was curved

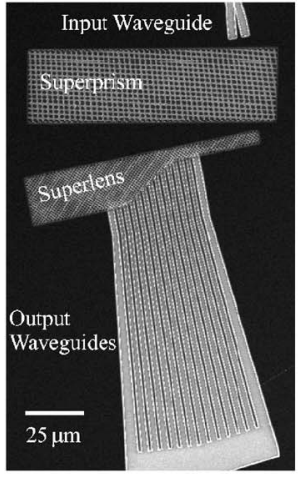

(a)

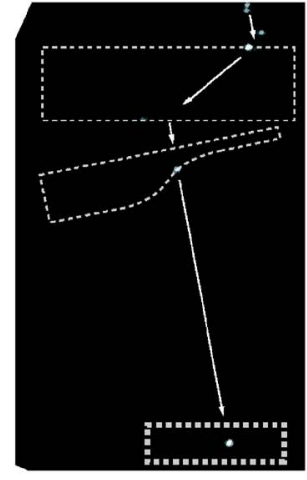

(b)

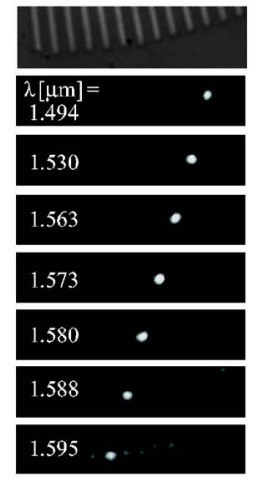

(c)

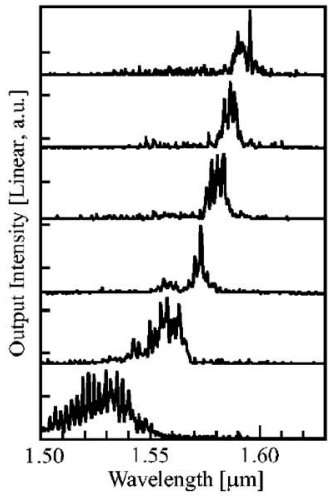

(d)
FIG. 3. (Color online) DEMUX device and its operation. (a) SEM image of fabricated device. (b) NFP of scattered light at $\lambda=1.37 \mu \mathrm{m}$ observed from above. (c) NFPs for seven different wavelengths at the end of output waveguides. The top figure shows an optical microscope image of the end of the output waveguides. (d) Demultiplexing action evaluated for six output waveguides. 
to compensate for chromatic aberration; the length of the superlens at each lateral position was equal to the focal length of the corresponding wavelength. The superlens was connected to 2 - $\mu \mathrm{m}$-wide output channel waveguides that were terminated by a rectangular hole for picking up the output light.

In the measurement, TE-polarized light having $\lambda$ $=1.50-1.63 \mu \mathrm{m}$ was used. Figures 3(b) and 3(c) show obtained NFPs. Weak light scattering was observed at the input and output surfaces of the PCs. However, the output light was much more intense than the scattered light, and was clearly shifted with the wavelength. Figure 3(d) shows transmission spectra obtained for six output waveguides. The oscillation in each spectrum was caused by the Fabry-Pérot resonance inside the output waveguide since the connection between the superlens and the output waveguide was not optimized and the output waveguide was simply terminated by a rectangular hole. This oscillation can be suppressed by introducing antireflection structures such as narrow tapered waveguides. The channel spacing of the four output waveguides on the longer wavelength side was $11 \mathrm{~nm}$; this is a narrow spacing for such a small device size (80 $\times 100 \mu \mathrm{m}^{2}$, excluding the input and output waveguides). Of course, it can be further narrowed by enlarging the superprism; a spacing of $0.4 \mathrm{~nm}$ has been theoretically predicted for a device size of $1 \mathrm{~mm}^{2} .{ }^{11}$ On the shorter wavelength side, the spectral width and channel spacing increased to 15-30 $\mathrm{nm}$ due to the lower angular dispersion. The channel spacing can be equalized by elongating the beam separation length of the superprism on the shorter wavelength side. The cross-talk between channels ranged from -6 to $-15 \mathrm{~dB}$. It will be reduced to less than $-20 \mathrm{~dB}$ by optimizing the position of output waveguides and reducing the internal resonance.

In conclusion, we fabricated PC slabs on a SOI substrate having an interface structures that minimize reflection and scattering losses. Using these, we were able to clearly observe negative refraction of light using the light cone condition. In the PC superprism, the angular dispersion $\Delta \theta / \Delta \lambda$ was measured to be $15^{\circ} / 60 \mathrm{~nm}$, which agrees with the FDTD simulation prediction. Demultiplexing was confirmed for a wavelength DEMUX device consisting of a PC superprism and a superlens. The channel spacing was $11 \mathrm{~nm}$ for an effective device size of $80 \times 100 \mu \mathrm{m}^{2}$. It is intended to achieve equal channel spacing and to suppress Fabry-Pérot resonance by further optimizing the structure.

This work was supported by The Core Research for Evolutional Science and Technology (CREST) Project of Japan Science and Technology Agency, International Communications Foundation, and Tateisi Science and Technology Foundation.

${ }^{1}$ H. Kosaka, T. Kawashima, A. Tomita, M. Notomi, T. Tamamura, T. Sato, and S. Kawakami, Phys. Rev. B 58, 10096 (1998).

${ }^{2}$ T. Baba and M. Nakamura, IEEE J. Quantum Electron. 38, 909 (2002).

${ }^{3}$ L. Wu, M. Mazilu, T. Karle, and T. F. Krauss, IEEE J. Quantum Electron. 38, 915 (2002).

${ }^{4}$ K. B. Chung and S. W. Hong, Appl. Phys. Lett. 81, 1549 (2002).

${ }^{5}$ T. Prasad, V. Colvin, and D. Mittleman, Phys. Rev. B 67, 165103 (2003).

${ }^{6}$ D. Scrymgeour, N. Malkova, S. Kim, and V. Gopalan, Appl. Phys. Lett. 82, 3176 (2003).

${ }^{7}$ A. I. Cabuz, E. Centeno, and D. Cassagne, Appl. Phys. Lett. 84, 2031 (2004).

${ }^{8}$ N. C. Panoiu, M. Bahl, and R. M. Osgood, Jr., J. Opt. Soc. Am. B 21, 1500 (2004).

${ }^{9}$ A. Lupu, E. Cassan, S. Laval, L. El Melhaoui, P. Lyan, and J. M. Fedeli, Opt. Express 12, 5690 (2004).

${ }^{10}$ T. Baba and T. Matsumoto, Appl. Phys. Lett. 81, 2325 (2002).

${ }^{11}$ T. Matsumoto, S. Fujita, and T. Baba, Opt. Express 13, 10768 (2005).

${ }^{12}$ H. Kosaka, T. Kawashima, A. Tomita, M. Notomi, T. Tamamura, T. Sato, and S. Kawakami, Appl. Phys. Lett. 74, 1212 (1999).

${ }^{13}$ M. Notomi, Phys. Rev. B 62, 10696 (2000).

${ }^{14}$ C. Luo, S. G. Johnson, J. D. Joannopoulos, and J. B. Pendry, Phys. Rev. B 68, 045115 (2003).

${ }^{15}$ P. V. Parimi, W. T. Lu, P. Vodo, and S. Sridhar, Nature (London) 426, 404 (2003).

${ }^{16}$ E. Cubukcu, K. Aydin, and E. Ozbay, Phys. Rev. Lett. 91, 207401 (2003).

${ }^{17}$ A. Berrier, M. Mulot, M. Swillo, M. Qiu, L. Thulen, A. Talneau, and S. Anand, Phys. Rev. Lett. 93, 073902 (2004).

${ }^{18}$ A. Husakou and J. Herrmann, Opt. Express 12, 6491 (2004).

${ }^{19}$ X. Wang, Z. F. Ren, and K. Kempa, Appl. Phys. Lett. 86, 061105 (2005).

${ }^{20}$ Z. Tang, R. Peng, D. Fan, S. Wen, H. Zhang, and L. Qian, Opt. Express 13, 9796 (2005)

${ }^{21}$ X. Zhang, Phys. Rev. B 71, 165116 (2005).

${ }^{22}$ M. Ke, Z. Liu, C. Qiu, W. Wang, J. Shi, W. Wen, and P. Sheng, Phys. Rev. B 72, 064306 (2005).

${ }^{23}$ E. Schonbrun, T. Yamashita, W. Park, and C. J. Summers, Phys. Rev. B 73, 195117 (2006).

${ }^{24}$ T. Matsumoto, K. Eom, and T. Baba, Opt. Lett. 31, 2776 (2006).

${ }^{25}$ B. Momeni, J. Huang, M. Soltani, M. Askari, S. Mohammadi, M. Rakhshandehroo, and A. Adibi, Opt. Express 14, 2413 (2006).

${ }^{26}$ T. Baba, T. Matsumoto, and M. Echizen, Opt. Express 12, 4608 (2004). 